\title{
Dissolved Gases in Oil Diagnosis Based on Support Vector Machine
}

\author{
Sun Guoxia ${ }^{1,2, a}$, Wu Haijiang ${ }^{1, a}$, Chen Guojun ${ }^{1, a}$, Ma Cuntao ${ }^{2, b}$ \\ ${ }^{1}$ Foshan Power Supply Bureau Guangdong Power Grid Corp.Foshan, China \\ ${ }^{2}$ School of Electrical Engineering Wuhan University Wuhan, China
}

Keywords: SVM ; transformer ; DGA ; fault

\begin{abstract}
A kind of analysis method in which SVM is used for power transformer DGA is proposed in this paper. This method uses the SVM algorithm to classify the composition of DGA in transformer and diagnoses the fault of the transformer. At the same time, it introduces the fuzzy membership function, and it can eliminate unable diagnosis area when the discrete decision function is used. Then by using a example to test this method, it shows the SVM play excellent performance in the fault diagnosis of power transformer.
\end{abstract}

\section{Introduction}

SVM is put forward by Vapnik in the 1990s,it's a new type of machine learning algorithm, which is built in strict statistical learning theory based on the structural risk minimization rule to gain real risk, effectively improve the algorithm generalization ability, it's a better solution to practical problems like small sample, nonlinear and high dimension in the past study methods, at the same time it overcomes the difficult in determine the network structure, the slow convergence, the small local point, over learning and less learning, and training required a large number of data sample etc. in the neural network learning method. Therefore, supporting vector machine technology provides a new solution for the field of electrical engineering intelligence.

For the Power transformer is the key equipment in the power system, the fault analysis has become an important topic in power system operation. The traditional analysis method of power transformer is the use of three-ratio method to analyze the composition of dissolved gases in oil, its application is unsatisfactory. This paper describes the basic principles of SVM, training methods and model selection, use SVM to the transformer fault diagnosis, research the SVM diagnosis methods of oil gas .

\section{SVM}

The basic idea of SVM is that mapped the sample space to the feature space by a kernel function (inner product function), and then find the optimal surface of the original sample in the feature space to get the nonlinear relationship between input variables and output variables, and find SVM for pattern classification, the computational complexity is almost no increase, the basic structure is shown in Figure 1.




SVM have a rigorous theoretical foundation, which is structural risk minimization principle, has a strong outreach capacity; SVC algorithm is a convex quadratic optimization problem to ensure that found the global optimal solution; can better solve the practical problems of the small sample, nonlinear, high dimension, etc., so that it can use as few samples as possible to get optimal classification surface to predict and then get $t$ the minimal cost ,so it can be obtained the best promotion.

for the analysis of gases dissolved in transformer oil, using the multi-class support vector machine classifier has a good analysis results. The basic principle of multi-class support vector machine classifier is as follows:

A given training set:

$T=\left\{\left(x_{1}, y_{1}\right), \cdots\left(x_{m}, y_{m}\right)\right\}$

Among, $x_{i} \in X=R^{m}, y_{i} \in Y=(1,2, \cdots, n)$,M is the training sample number, $\mathrm{m}$ is the number of input variables, $\mathrm{n}$ is the number of categories. Deal with multi-classification problem is to find a decision function, make

$$
f(x): X=R^{m} \rightarrow Y \text {. (1) }
$$

Generally speaking, there are four ways of support vector machine multi-classification problem:(1) one-to-many;(2) one-against-one method(one-to-one);(3) error-correcting output codes;(4) One-off Solution. As the space is limited, this section only explains the classification of pairs.

One-against-one is a classification method based on binary problems, it can reduce the unclassifiable regions of one-to-many classification .The specific method is "one-to-one method", that is, in the n-class, select two different categories, namely constitute an SVM sub-classifiers, so that a total of $n(n-1) / 2$ sub-classifiers. When constructed the SVM sub-classifier of category I and category $\mathrm{j}$, selected in the sample data sets belong to the class $\mathrm{i}$ and the sample data of category as training sample data, and the data belongs to category $i$ is marked as positive, the data belongs to category $\mathrm{j}$ is marked as negative. Set the optimization decision function of category $\mathrm{i}$ and category $\mathrm{j}$ for $D_{i j}(x)=W_{i j}^{T} g(x)+b_{i j}$, In the expression $W_{i j}$ is l-dimensional vector, $g(x)$ is the mapping function, $b_{i j}$ is bias term. And $D_{i j}(x)=-D_{j i}(x)$.The regional of pairs algorithm $R_{i}=\{x \mid$ $\left.D_{i j}(x)>o, j=1, \cdots . n, j \neq i\right\}$ no longer overlap. If $\mathrm{x}$ is located within the $\mathrm{R}_{\mathrm{i}}$, it will be into the category i. If $x$ is located within the $R_{i}$, we can determine the category of $x$ in the referendum law. The specific approach: First calculate the

$$
D_{i}(x)=\sum_{j \neq i, j=1}^{n} \operatorname{sign}\left[D_{i j}(x)\right]
$$

In the expression, $\operatorname{sign}(x)= \begin{cases}1, & x \geq 0 \\ -1 & x<0\end{cases}$

Then, in accordance with the following formula decided the category of $\mathrm{x}$, that is

$$
\arg \max _{i=1 \cdots k} D_{i}(x)
$$

\section{Diagnostic method for support vector machines}

A. The establishment of the diagnostic model of support vector machines

The power transformer fault diagnosis can be understood as a multi-class classification problem, shown in table 1:

\begin{tabular}{|l|c|c|c|c|c|c|}
\hline $\begin{array}{c}\text { Terial } \\
\text { number }\end{array}$ & $\mathbf{1}$ & $\mathbf{2}$ & $\mathbf{3}$ & $\mathbf{4}$ & $\mathbf{5}$ & $\mathbf{6}$ \\
\hline Property & Low & High & Low- & High- & High- & Health \\
\hline
\end{tabular}




\begin{tabular}{|l|l|l|l|l|l|l|}
\hline & $\begin{array}{l}\text { Temperature } \\
\text { Superheater }\end{array}$ & $\begin{array}{l}\text { temperature } \\
\text { overheating }\end{array}$ & $\begin{array}{l}\text { energy } \\
\text { discharge }\end{array}$ & $\begin{array}{l}\text { energy } \\
\text { discharge }\end{array}$ & $\begin{array}{l}\text { energy } \\
\text { discharge } \\
\text { and Chief } \\
\text { overheating }\end{array}$ & \\
\hline
\end{tabular}

For classification problems, SVM algorithm itself is a second-class classifier. For K-class problem, one-to-many dissemination need to construct K SVM sub-classifier, the i-th of the SVM classifier separated $i$-th class from the other classes. One-to-one algorithm will need to construct $1 / 2 \mathrm{~K}$ (K-1) SVM classifier, The former need to construct SVM sub-classifier is much less. However, one or several algorithms in the training process, the number of training samples is only the current two categories corresponding to the former of training samples. The training sample number is large, and the training time is very long. However, one-on-one algorithm cost shorter time. Therefore, this paper mainly uses one-on-one algorithm to construct the power transformer fault diagnosis model, it has a total of five categories of failure, and need to construct $1 / 2 \mathrm{k}(\mathrm{k}-1)=10$ sub-classifiers.

SVM consists of 10 sub-SVM classifier, taking into account many sub-SVM model, in order to accelerate the training process, select the least squares support vector machine, kernel function directly use the RBF kernel, $\mathrm{C}=6.25$. In order to facilitate comparison with the three-ratio method and neural networks, data processing selects five gases $\mathrm{H}_{2}, \mathrm{CH}_{4}, \mathrm{C}_{2} \mathrm{H}_{2}, \mathrm{C}_{2} \mathrm{H}_{4}, \mathrm{C}_{2} \mathrm{H}_{6}$ as the input of text samples. And still use the value method to deal with the DGA original data "normalized".

B. SVM training

1) The first layer of the SVM training

The SVM is a two-classifier, the selection of L1 soft margin support vector machine, in the training process, taking the dangers of the fault condition is mistaken for health is far greater than the health status mistaken for a fault condition. In addition, the number of healthy samples in the training sample is much smaller than the number of fault samples, to overcome this imbalance problem, marginal coefficient for two types of data set, set the normal state of the $\mathrm{c}+$, the fault status is set to $\mathrm{c}-$, and $\mathrm{c}->\mathrm{c}+$.

To determine the kernel function for the RBF kernel coefficient, $\mathrm{C}+1.25, \mathrm{C}-=12.5$, $\sigma^{2}=1.25$.Now the training and testing accuracy were $100 \%$. Trained to construct diagnostic decision-making function are as follows:

$$
D(x)=\sum_{i \in S} \alpha_{i} y_{i} H\left(x_{i}, x\right)+b
$$

Diagnostic methods for:

$\left\{\begin{array}{cc}\text { healthy } & \text { ifD }(\mathrm{x})>0 \\ \text { fault } & \text { ifD }(\mathrm{x})<0\end{array}\right.$

fault ifD $(\mathrm{x})<0$

2) The second layer of the SVM training

The second layer SVM include 10 sub-SVM classifier, taking into account the more sub-SVM model, in order to speed up the training process, the selection of least squares support vector machine algorithm. Kernel function selection of the RBF kernel, $\mathrm{C}=6.25, \sigma^{2}=1.25$

C. The synthesis of sub-SVM classifier results

After the completion of all 10 sub-SVM classifier training, 10 classification decision function can be obtained, and the function of category I and category $\mathrm{j}$ is:

$$
D_{i j}(x)=\sum_{i \in S} \alpha_{i} y_{i} H\left(x_{i}, x\right)+b_{i j}
$$

And have the sample

$D_{i j}(x)=-D_{j i}(x)$

Be calculated for different types of decision-making function for the sample $\mathrm{x}$

$D_{i}(x)=\sum_{j \neq i, j=1}^{k} \operatorname{sign}\left[D_{i j}(x)\right]$

In the expression, $\operatorname{sign}(a)= \begin{cases}1, & a>0 \\ 0 & \text { others }\end{cases}$ 
The diagnosis result of the sample of $\mathrm{x}$ is :

$$
\arg \max _{i=1 \cdots k} D_{i}(x)
$$

If only class i satisfies(7), the diagnosis results is the category $\mathrm{i}$; However, if there are multiple categories satisfy the equation (7).To solve this problem, the introduction of the fuzzy membership function $m_{i j}(x)$, defined as follows:

$$
m_{i j}(x)=\left\{\begin{array}{cc}
1 & D_{i j}(x) \geq 1 \\
D_{i j(x)} & \text { others }
\end{array}\right.
$$

Fuzzy membership function of the sample of $x$ for class $i$ is:

$$
m_{i}(x)=\min _{j=1 \cdots k} m_{i j}(x)(9)
$$

$\mathrm{T}$ he diagnosis result of the sample of $\mathrm{x}$ is:

$$
\arg \max _{j=1 \cdots k} m_{i}(x)(10)
$$

It can be found that the introduction of the fuzzy membership function can eliminate the nondiagnostic district of discrete decision function.

\section{Instance validation and results analysis}

After the end of the training process, the test sample of Appendix Table 1 is used to set the model for testing. Test results are shown in Table2.

Table 2 double-layer SVM model diagnosis

\begin{tabular}{|l|l|l|l|l|l|l|}
\hline \multicolumn{1}{|c|}{$\begin{array}{c}\text { diagnosis } \rightarrow \\
\text { actual failure } \downarrow\end{array}$} & health & $\begin{array}{c}\text { Low } \\
\text { Temperature } \\
\text { Superheater }\end{array}$ & $\begin{array}{c}\text { hyperthermia } \\
\text { and } \\
\text { superheating }\end{array}$ & $\begin{array}{l}\text { Low energy } \\
\text { discharge }\end{array}$ & $\begin{array}{c}\text { High energy } \\
\text { discharge }\end{array}$ & $\begin{array}{c}\text { High } \\
\text { let } \\
\text { heat }\end{array}$ \\
\hline health(1) & 1 & 0 & 0 & 0 & 0 & 0 \\
\hline $\begin{array}{l}\text { Low Temperature } \\
\text { Superheater (3) }\end{array}$ & 0 & 3 & 0 & 0 & 0 & 0 \\
\hline $\begin{array}{l}\text { hyperthermia and } \\
\text { superheating (2) }\end{array}$ & 0 & 0 & 2 & 0 & 0 & 0 \\
\hline $\begin{array}{l}\text { Low en } \\
\text { discharge (1) engy }\end{array}$ & 0 & 0 & 0 & 1 & 0 & 0 \\
\hline $\begin{array}{l}\text { High } \\
\text { discharge (3) energy }\end{array}$ & 0 & 0 & 0 & 0 & 3 & 0 \\
\hline High let heat (2) & 0 & 0 & 0 & 0 & 1 & 1 \\
\hline
\end{tabular}

Analysis in Table 2, the following conclusions:

(1) All fault conditions are correct diagnosis, failure misdiagnosed as healthy.

(2) In the diagnostic process of the specific fault class, only one case of high-level radioactive Chief overheating mistakenly diagnosed as high-energy discharge

The whole diagnostic accuracy of up to $91.67 \%$, the effect is satisfactory.

\section{Conclusion}

SVM (support vector machine) is a new algorithm, emerging in the field of machine learning. Compared with the classic machine learning method, the neural network, it has features as follows:(1)SVM, depriving from statistical learning theory, is specially aimed at situations of limited samples, with the goal of getting the optimal solution via the existing information but not just obtaining the optimal value under infinite samples.(2)SVM, with the seeking of minimal structured risk, will be able to minimize the empirical risk and fiducially range .(3)Support vector machines (SVM) finally turns into a quadratic optimization problem which, in theory, could achieve the global optimization, solving the inevitable local extremer problems in neural network method. The topology structure of SVM is decided by support vectors, which can be obtained through the optimizing process, overcoming the difficulty in choosing the neural network structure. This paper 
realizes dissolved gas analysis with the SVM algorithm. It achieves good results, with different gas composition categorized and the transformer breakdown type obtained.

\section{References}

[1] Ni Jian.Dianosis of Insidious Fault Inside Power Transformer [J]. High voltage electrical Journal,2001,37(3):54-55

[2] IEC60599.Mineral oil-impregnated electrical equipment in service guide to the interpretation of dissolved and free gases analysis, second edition.1999

[3] Zhang Y.An artificial new network approach to transformer fault diagnosis[J].IEEE Transactions on Power Delivery, 1996,11(4) :1836-1841

[4] Wang Dazhong ,Xu Wen ,Zhou Zecun.Application of Fuzzy Sets Theory,Expert System and Artifical Neural Networks in Faults Diagnosis of Power Transformer[J]. Chinese Journal of Mechanical Engineering ,1996,16(5):349-353

[5] Lin CE,Ling J M,Huang C L.An expert system for transformer fault diagnosis using Dissolved gas analysis[J].IEEETransactions on PowerDelivery,1993,8(1):231-238

[6] Wang Z Y.A combined ANN and expert system tool for transformer fault diagnosis[J].IEEE Transactions on Power Delivery,1998,13(4):1224-1229

[7] Chow $M$ et al.On the application and design of artificial neural networks for motor fault detection, IEEE Trans.Ind.Electron, 1991,38(6):448-453

[8] Sun Xincai,Chen Weigen,Li Jian.Electrical Equipment in Oil Gas Online Monitoring and Fault Diagnosis Technology[M].Bei Jing: Science and Technology Press,2003

[9] Rui Ming.Theory of Support Vector Machine and Its Application Analysis[M].Bei Jing: China Electric Power Press.

Appendix:

Table 1 the test sample set

\begin{tabular}{|c|l|c|c|c|c|c|}
\hline $\begin{array}{c}\text { Serial } \\
\text { number }\end{array}$ & \multicolumn{1}{|c|}{ Actual failure } & \multicolumn{1}{|c|}{$\mathrm{H}_{2}$} & $\mathrm{CH}_{4}$ & $\mathrm{C}_{2} \mathrm{H}_{6}$ & \multicolumn{2}{c|}{$\mathrm{C}_{2} \mathrm{H}_{4}$} \\
\hline 1 & Low temperature overheating & 120 & 120 & 3.3 & 84 & 0.55 \\
\hline 2 & Low temperature overheating & 93 & 58 & 43 & 37 & 0 \\
\hline 3 & Low temperature overheating & 160 & 130 & 33 & 96 & 0 \\
\hline 4 & High temperature overheating & 98 & 123 & 33 & 2.6 & 16 \\
\hline 5 & High temperature overheating & 73 & 520 & 140 & 120 & 6 \\
\hline 6 & Low-energy discharge & 1565 & 93 & 34 & 47 & 0 \\
\hline 7 & High-energy discharge & 150 & 27 & 5.6 & 65 & 90 \\
\hline 8 & High-energy discharge & 32.4 & 5.5 & 1.4 & 12.6 & 13.2 \\
\hline 9 & High-energy discharge & 335 & 67 & 18 & 143 & 170 \\
\hline 10 & $\begin{array}{l}\text { High-energy discharge and Chief } \\
\text { overheating }\end{array}$ & 200 & 14 & 117 & 131 & 5.6 \\
\hline 11 & $\begin{array}{l}\text { High-energy discharge and Chief } \\
\text { overheating }\end{array}$ & 59 & 28 & 9 & 70 & 15 \\
\hline 12 & Health & 46 & 11.5 & 33 & 8.5 & 0.6 \\
\hline
\end{tabular}

